\title{
ИССЛЕДОВАНИЕ ОТКАЗОУСТОЙЧИВОГО ВЕНТИЛЬНО-ИНДУКТОРНОГО ЭЛЕКТРОДВИГАТЕЛЯ НАСОСА ДЛЯ ДОБЫЧИ НЕФТИ
}

\author{
Однокопылов Георгий Иванович', \\ OGlz@yandex.ru
}

Букреев Виктор Григорьевич',

bukreev@tpu.ru

\author{
Розаев Иван Андреевич', \\ veazor@tpu.ru
' Национальный исследовательский Томский политехнический университет, Россия, 634050, г. Томск, пр. Ленина, 30.

Актуальность. На сегодняшний день нефтедобыча является одним из приоритетных направлений промышленного развития в стране. В связи с этим важным является повышение эффективности работы электродвигателя, входящего в состав электротехнических комплексов установок нефтедобывающих и нефтеперекачивающих насосов. Именно этот элемент комплекса в значительной степени определяет надежность технологической системы в целом. В частности, аварийный выход из строя электродвигателя влечет за собой технологическую остановку производства и приводит к недоотпуску нефти и как следствие к значительным экономическим потерям. Поэтому обеспечение отказоустойчивости исполнительного электродвигателя является важнейшей задачей. Одним из вариантов решения такой задачи является использование перспективного вентильно-индукторного двигателя в совокупности с алгоритмами отказоустойчивого управления.

Цель: разработка алгоритмов отказоустойчивого управления исполнительным вентильно-индукторным электродвигателем в неполнофазных режимах работы; повышение качества функционирования вентильно-индукторного электродвигателя насоса в аварийных неполнофазных режимах работы путем подключения алгоритмов отказоустойчивого управления, позволяющих сохранять и полностью либо частично восстанавливать работоспособность исполнительного вентильно-индукторного электродвигателя при однократных и множественных отказах силовых цепей.

Объект: трехфазный вентильно-индукторный электродвигатель нефтедобывающего насоса в неполнофазных режимах работы. Методы: теория электрических машин; методы описания динамических электромеханических и магнитных процессов; математическое и имитационное моделирование, программирование в среде MathCad и Matlab Simulink.

Результаты. Разработана математическая и имитационная модели отказоустойчивого трехфазного вентильно-индукторного электропривода. Получены напорно-расходные характеристики нефтедобывающего насоса в неполнофазном режиме работы исполнительного вентильно-индукторного электродвигателя с применением алгоритмов отказоустойчивого управления.

\section{Ключевые слова:}

Вентильно-индукторный электродвигатель, обрыв фазы электродвигателя, отказоустойчивый электропривод, алгоритм отказоустойчивого управления, напорно-расходная характеристика насоса.

\section{Введение}

В настоящее время нефтедобыча занимает значительный экономический кластер энергетического сектора Российской Федерации. При этом эффективность разработки нефтегазовых месторождений в значительной степени определяется непрерывной диагностикой и оптимизацией эксплуатационных режимов системы «скважина-насос» $[1,2]$. Аварийный выход из строя исполнительного электродвигателя в составе установки насоса добычи нефти влечет за собой значительные экономические потери для нефтедобывающей организации [3].

С целью предотвращения аварийных отключений насоса добычи нефти предлагаются технические решения, которые позволяют продолжить работу электроцентробежного насоса (ЭЦН) после однократных или множественных отказов силовой части статорных обмоток исполнительного двигателя [4-6]. Существует ряд исследований, предлагающих внедрение вентильно-индукторного электродвигателя (ВИД) в качестве исполнительного двигателя насосного агрегата. Имеющиеся разработки касаются преимущественно конструктивных особенностей ВИД и его модернизации [7-9]. Однако на сегодняшний день ВИД не получил должного развития и серийного производства. Вентильно-индукторный электродвигатель обладает рядом преимуществ по сравнению с асинхронными и вентильными двигателями [10-13]. К достоинствам ВИД относятся: надежность и простота конструкции и технологии изготовления; возможность получать как сверхвысокие, так и сверхнизкие частоты вращения вала; гибкость системы управления; ротор ВИД имеет малый момент инерции, что позитивно отражается на динамике его работы; ВИД способен работать в тяжелых перегрузочных режимах и в широком диапазоне нагрузок; за счет электрически независимых фаз ВИД обладает исходной отказоустойчивостью; ВИД обладает высокими энергетическими характеристиками и КПД [8]. Следует отметить, что преимущества ВИД в полной мере приведены в работах [14-17]. 
Управление ВИД в рабочих режимах отличается от управления в аварийных режимах работы, так как обрыв фазы двигателя вызывает защитное отключение системы электропривода [18-21]. Сoответственно для управления электроприводом в аварийных и неполнофазных режимах работы необходимо разработать алгоритмы мониторинга и управления, отладку которых целесообразно проводить на основе моделирования [22-25].

На начальном этапе исследований был определен перечень объектов нефтяных скважин, моделирование которых является необходимым с точки зрения синтеза и разработки алгоритмов управления [26-29]. Установка нефтедобывающего или нефтеперекачивающего насоса представляет собой электротехнический комплекс, состоящий из системы алгоритмического управления (СУ), исполнительного вентильно-индукторного электродвигателя, центробежного насоса (ЦН), блока обработки телеметрических данных (БТД). В связи с этим предложенная модель построена объединением моделей ВИД, ЦН, СУ с моделью, учитывающей потери напряжения в кабельной линии (КЛ). При этом модели движения жидкости не рассматривались, учитывалось только влияние среды на формирование момента нагрузки [30,31].

\section{Построение математической модели ВИд}

При создании математической модели исследуемого ВИД приняты следующие допущения: ВИД обладает электрической и магнитной симметрией; значения активных сопротивлений фаз электродвигателя равны; ключи силового преобразователя приняты идеальными, мощность источника питания принимается значительно большей по сравнению с мощностью двигателя, запаздывания в СУ отсутствуют, влияние добавочных вихревых токов и гистерезиса в магнитопроводе учитывается при моделировании алгоритмов с изменением углов перекрытия при изменении частоты питающей сети. В основу математического описания ВИД положены уравнения электрического равновесия фаз двигателя и уравнения формирования электромагнитного момента [22].

Матричное уравнение равновесия напряжений в фазах трехфазного ВИД в нормальной форме Коши имеет вид (1):

$$
\frac{d[i]}{d t}=\left[L_{d}\right]^{-1}\left\{[U]-[R][i]-\omega\left[K_{\omega}\right]\right\},
$$

где [i] - матрица неизвестных (токи фаз статора ВИД); [U] - матрица напряжений фаз ВИД; $[R]-$ матрица сопротивлений фаз ВИД; $\left[L_{d}\right]-$ прямая матрица дифференциальных индуктивностей фаз ВИД в функции фазных токов и текущего положения ротора; $\left[K_{\omega}\right]$ - матрицы коэффициентов противо-ЭДС фаз ВИД в функции фазных токов и текущего положения ротора; $\omega$ - угловая скорость вращения ротора.

Представим каждый элемент уравнения (1) отдельной матрицей:

$$
\begin{gathered}
{[i]=\left[\begin{array}{l}
i_{A} \\
i_{B} \\
i_{C}
\end{array}\right] ;[U]=\left[\begin{array}{l}
U_{A} \\
U_{B} \\
U_{C}
\end{array}\right] ;[R]=\left[\begin{array}{ccc}
R_{s} & 0 & 0 \\
0 & R_{s} & 0 \\
0 & 0 & R_{s}
\end{array}\right] ;} \\
{\left[L_{d}\right]=\left[\begin{array}{ccc}
L_{A}\left(i_{A}, \theta\right) & 0 & 0 \\
0 & L_{B}\left(i_{B}, \theta\right) & 0 \\
0 & 0 & L_{C}\left(i_{C}, \theta\right)
\end{array}\right] ;} \\
{\left[K_{\omega}\right]=\left[\begin{array}{ccc}
K_{A}\left(i_{A}, \theta\right) & 0 & 0 \\
0 & K_{B}\left(i_{B}, \theta\right) & 0 \\
0 & 0 & K_{C}\left(i_{C}, \theta\right)
\end{array}\right] .}
\end{gathered}
$$

При моделировании аварийных и неполнофазных режимов работы ВИД необходимо в состав уравнений равновесия напряжений в фазах (1) ввести матрицу $\left[M_{0}\right]$ отказов (в общем случае полученную на основе работы алгоритма мониторинга). Это позволит имитировать отказ типа «обрыв фазы» или «не включение/не выключение ключа преобразователя частоты»:

$$
\left[M_{0}\right]=\left[\begin{array}{ccc}
\bar{a} & 0 & 0 \\
0 & \bar{b} & 0 \\
0 & 0 & \bar{c}
\end{array}\right] .
$$

При этом единичному состоянию битов отказов $a, b, c$ будет соответствовать нормальный полнофазный режим работы, а нулевому - аварийный режим работы при обрыве соответствующей фазы обмотки статора [24].

Полное выражение для матрицы активных сопротивлений в нормальной форме Коши принимает следующий вид:

$$
\left[R_{M}\right]=\left([1]+k_{\infty}\left[M_{0}\right]\right)[R],
$$

где $k_{\infty}$ - коэффициент, имитирующий введение высокоомного сопротивления в цепь статора. В этом случае матричное уравнение равновесия напряжений в фазах трехфазного ВИД с учетом матрицы отказов $\left[R_{M}\right]$ в нормальной форме Коши записывается уравнением:

$$
\frac{d[i]}{d t}=\left[L_{d}\right]^{-1}\left\{[U]-\left[R_{M}\right][i]-\omega\left[K_{\omega}\right]\right\} .
$$

Преобразование магнитной энергии в механическую происходит при постоянстве потокосцепления и связано с изменением угла поворота ротора и магнитной проводимости в воздушном зазоре. Следовательно, при рассмотрении вращающейся электрической машины зависимость механической энергии от электромеханического момента при изменении положения ротора $n$-й фазы ВИД записывается выражением [32, 33]:

$$
\Delta W_{n}=M_{n} \cdot \Delta \theta,
$$

где $M_{n}$ - электромагнитный момент двигателя, формируемый $n$-й фазой; $\Delta W_{n}$ - изменение электромеханической энергии, формируемой $n$-й фазой на интервале $\Delta \theta$ угла поворота ротора [32]. 
Запишем уравнение для электромагнитного момента через производную потокосцепления по углу поворота ротора, которое для $n$-й фазы имеет вид [32-34]:

$$
M_{n}=i_{n} \frac{\partial \Psi_{n}, \theta_{n}}{\partial \theta}
$$

Приняв $K_{\omega n}=\frac{\partial \Psi\left(i_{n}, \theta_{n}\right)}{\partial \theta}$, данное уравнение записывается более компактно:

$$
M_{n}=i_{n} \cdot K_{\omega n} .
$$

Мгновенный электромагнитный момент ВИД представляет собой сумму моментов, формируемых подключенными фазами двигателя (6). С учетом принятых допущений запишем суммарный момент $M$ трехфазного ВИД [22], [32]:

$$
M=M_{A}\left(i_{A}, \theta_{n}\right)+M_{B}\left(i_{B}, \theta_{n}\right)+M_{C}\left(i_{C}, \theta_{n}\right),
$$

где $M_{A}\left(i_{A}, \theta_{n}\right), M_{B}\left(i_{B}, \theta_{n}\right), M_{C}\left(i_{c}, \theta_{n}\right)$ - моменты, развиваемые фазами $A, B, C$ трехфазного двигателя. Уравнение движения электропривода можно представить в виде:

$$
\frac{d \omega}{d t}=\frac{p}{J}\left(M-M_{\mathrm{c}}\right),
$$

где $J$ - суммарный момент инерции электропривода; $p$ - число пар полюсов; $\omega$ - угловая скорость вращения ротора; $M_{\mathrm{c}}$ - момент сопротивления.

\section{Математическое и имитационное моделирование ЭЦН с ВИД}

Состояние ЦН определяется такими параметрами, как: $Q$ - объемный расход жидкости, проходящей через ЦН; $P$ - давление жидкости на выходе ЦН. Основным параметром КЛ, влияющим на напряжение, поступающее на вход электродвигателя, является волновое сопротивление $Z$ кабеля. Которое определяется отношением напряжения падающей волны к току этой волны в линии передачи. Структура модели ЭЦН с ВИД показана на рис. 1.

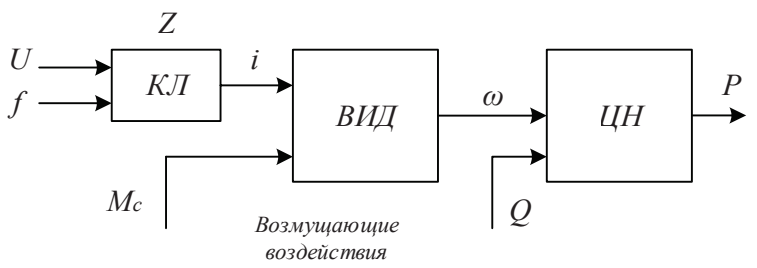

Pис. 1. Структура модели насоса добычи нефти

Fig. 1. Installation of the centrifugal pump model structure

Статическая модель центробежного насоса формируется на основе напорно-расходной характеристики насоса, которая аппроксимируется зависимостью [35]:

$$
H_{\mathrm{H}}=H_{f \mathrm{H}}\left(\frac{\omega}{\omega_{\mathrm{H}}}\right)^{2}+S_{f} Q^{2},
$$

где $\omega_{\text {н }}$ - номинальное значение частоты вращения вала насоса; $H_{\text {н }}$ у уровень напора на выходе насоса; $H_{f н}-$ принятое «фиктивное» значение напора насоса на номинальной частоте вращения; $S_{f}-$ принятое значение гидравлического сопротивления насоса. Далее, выразив напор через давление, получено:

$$
H=\frac{P}{\rho g},
$$

где $\rho$ - значение плотности протекаемой жидкости, $g$ - ускорение свободного падения, полученное уравнение статической модели ЦН примет вид:

$$
\frac{P}{\rho g}-H_{f_{\mathrm{H}}}\left(\frac{\omega}{\omega_{\mathrm{H}}}\right)^{2}+S_{f} Q^{2}=0 .
$$

Постоянная времени, определяющая инерцию изменения давления, уменьшается пропорционально снижению расхода и давления в скважине и не сказывается на системе управления в рассматриваемых условиях. Поэтому переходными процессами в жидкости и инерционностью ЦН в данной модели предлагается пренебречь $[35,36]$. Момент от сил трения на валу электродвигателя можно принять постоянным и равным $\Delta M_{\text {стр }}$. Момент сопротивления жидкости $\Delta M_{\text {с ж }}$ принимается равным постоянному значению с учетом характера жидкости, условий эксплуатации, глубины скважины и вязкости [30], [35]. Для механизма с вентиляторным типом нагрузки, что характерно для насоса, статический момент сопротивления на валу двигателя изменяется в зависимости от скорости вращения электродвигателя в соответствии с выражением (9) [35-37]:

$$
M_{\mathrm{c}}(\omega)=M+\left(M_{\mathrm{c} \text { max }}-\Delta M_{\text {с тр }}-\Delta M_{\text {сж }}\right)\left(\frac{\omega}{\omega_{\mathrm{H}}}\right)^{2} \text {. }
$$

Таким образом, математическая модель ЭЦН на базе ВИД (10) может быть получена из уравнений (1)-(9) [22], [32], [35]:

$$
\left\{\begin{array}{c}
\frac{d[i]}{d t}=\left[L_{d}\right]^{-1}\left\{[U]-\left[R_{\mathrm{M}}\right][i]-\omega\left[K_{\omega}\right]\right\} ; \\
{\left[R_{\mathrm{M}}\right]=\left([1]+k_{\infty}\left[M_{\mathrm{o}}\right]\right)[R] ;} \\
\frac{d \omega}{d t}=\frac{p}{J}\left(M-M_{\mathrm{c}}\right) ; \\
M=M_{A}\left(i_{A}, \theta_{n}\right)+M_{B}\left(i_{B}, \theta_{n}\right)+M_{C}\left(i_{C}, \theta_{n}\right) ; \\
M_{\mathrm{c}}(\omega)=M+\left(M_{\mathrm{c} \max }-\Delta M_{\mathrm{c} \mathrm{тр}}-\Delta M_{\mathrm{c}}\right)\left(\frac{\omega}{\omega_{\mathrm{H}}}\right)^{x} ; \\
\frac{P}{\rho g}-H_{f \mathrm{H}}\left(\frac{\omega}{\omega_{\mathrm{H}}}\right)^{x}+S_{f} \cdot Q^{2}=0 ; \\
\theta_{n}=p \int_{0}^{t} \omega d t=p \theta .
\end{array}\right.
$$

Основные принципы построения модели ВИД в среде имитационного моделирования MatLab Si- 
mulink рассмотрены в ряде работ [38-41]. Структурная схема имитационной модели ЭЦН с ВИД показана на рис. 2.

Строение блоков «возмущающее воздействие

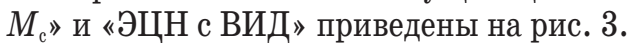

Описание устройства блоков преобразователя частоты (ПЧ), кабельной линии (КЛ), датчика положения (ДП) и блока регуляторов подробно рассмотрены в ранее опубликованных работах [22, 24, 25].

\section{Алгоритмы отказоустойчивого управления ВИД}

Рассмотрим алгоритмы отказоустойчивого управления вентильно-индукторным электроприводом. Использование исходной отказоустойчивости 3-фазного ВИД в аварийном 2-фазном режиме позволяет обеспечить живучесть с ограничением момента и частоты вращения на уровне $2 / 3$ от но- минального значения при отказах ПЧ или обрыве одной из обмоток статора [22].

Для упрощения моделирования и отображения алгоритмов датчик положения принят как «условный» датчик. Очевидно, что на сегодняшний день установки ЭЦН не оснащаются такими датчиками, так как невозможно обеспечить длительную работу такого датчика в условиях нефтедобывающей скважины и в следствие затруднений с передачей данных на поверхность с необходимой скоростью. В реальных электромеханических комплексах насосных агрегатов положение ротора электродвигателя определяется путем математических вычислений на основе мгновенных значений токов и напряжений $[42,43]$. Однако с точки зрения алгоритмов отказоустойчивого управления не имеет значения, как именно были получены данные о по-

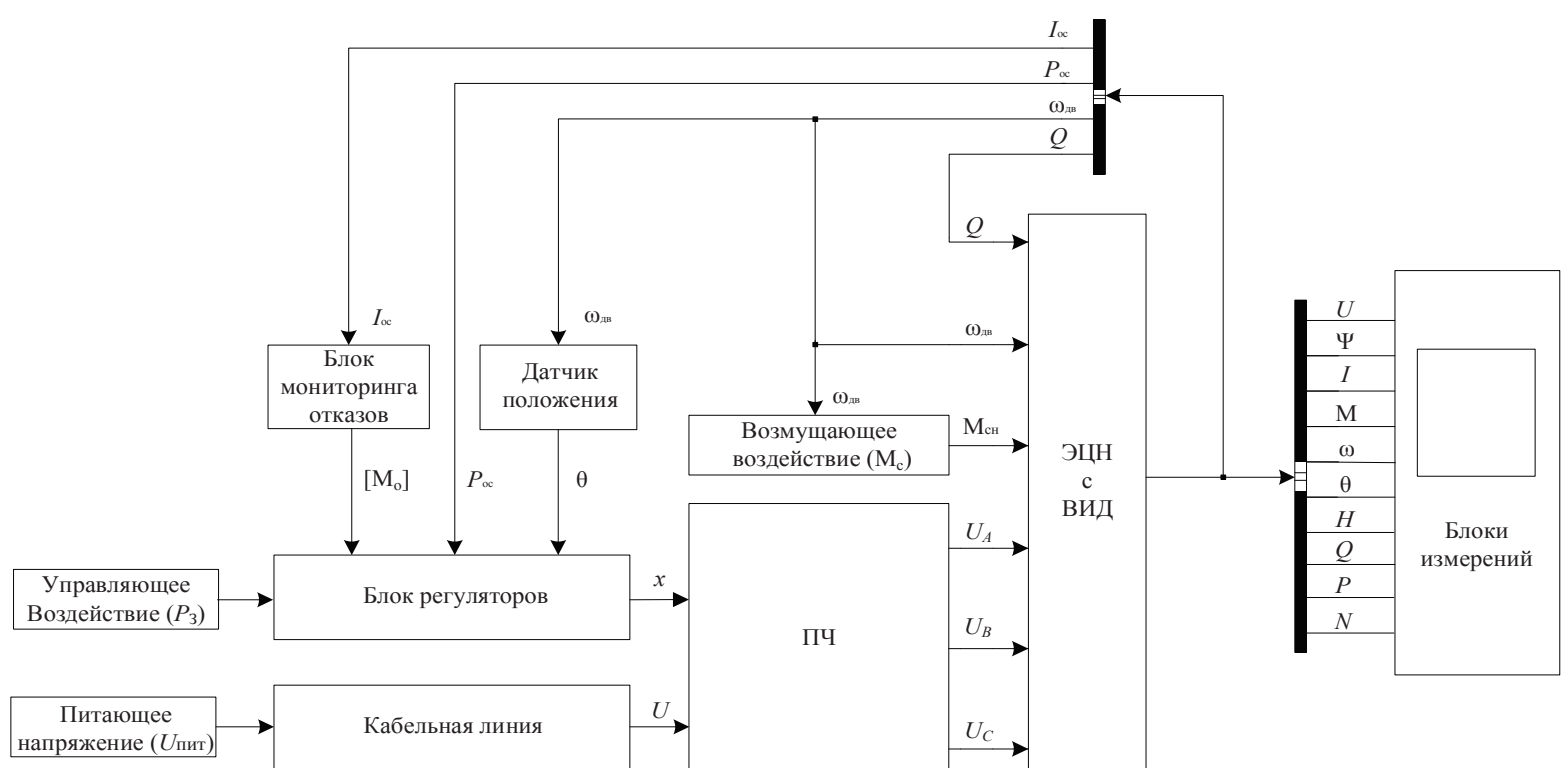

Pис. 2. Структурная схема илитационной модели установки ЭЦН с ВИД

Fig. 2. Functional block diagram of centrifugal pump installation with switched-reluctance motor simulation model

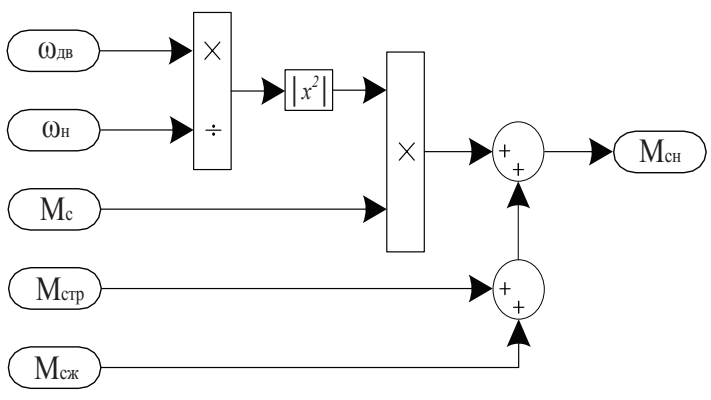

$a / a$



$\sigma / b$

Pис.3. а) блок «возмущающее воздействие $M_{c}$, б) блок «электроцентробежный насос с вентильно-индукторныл двигателем»

Fig. 3. a) block «perturbation influence $M_{c}$, b) block «centrifugal pump with switched-reluctance motor» 
ложении ротора, важным является дальнейшая работа с полученными данными. Аппаратное применение бездатчикового управления в погружных насосах с вентильным двигателем изучено и широко используется, например, группой компаний «Новомет» и в статье не рассматривалось [44].

Для обеспечения отказоустойчивости и увеличения функциональных возможностей при работе как на двух, так и на одной оставшейся в работе фазе электродвигателя было разработано и предложено три алгоритма.

1) Алгоритм управления трехфазным ВИД с симметричной одиночной коммутацией и компенсацией момента за счет увеличения амплитуды фазных токов. Из конструктивных особенностей ВИД следует, что все три фазы электрически независимы друг от друга и при отказе типа «обрыв фазы» можно компенсировать падение активной мощности за счет увеличения значений токов в оставшихся в работе фазах при условии наличия резерва по мощности (исходная нагрузка не более 66 \% от номинальной), чтобы избежать перегрева обмоток и обеспечить возможность продолжительного функционирования в неполнофазном режиме работы.

2) Алгоритм управления трехфазным ВИД с компенсацией момента за счет изменения угла перекрытия фаз. В общем случае управление ВИП осуществляется путем поочередной коммутации фаз двигателя на основании информации, получаемой от датчика положения ротора. Стандартной коммутацией принято считать симметричную одиночную коммутацию фаз, при которой перекрытие фаз отсутствует. С несимметричной одиночной коммутацией перекрытие фаз производится с углом перекрытия: $\theta_{\text {к }}=\pi / 4$. При активизации алгоритма восстановления происходит смена режима коммутации с симметричной одиночной в трехфазном режиме на несимметричную одиночную в неполнофазном режиме [22], [24].

3) Алгоритм управления трехфазным ВИД с компенсацией момента за счет изменения угла перекрытия фаз и увеличения амплитуд фазных токов. При активизации алгоритма восстановления происходит смена режима коммутации с симметричной одиночной на несимметричную одиночную с одновременным увеличением амплитуд фазных токов в 1,5 раза.

Функционирование ВИД с любым из представленных алгоритмов отказоустойчивого управления (1)-(3) подразумевает работу в кратковременном режиме с дальнейшим переходом на режим работы с симметричной одиночной коммутацией и исходной отказоустойчивостью с восстановлением частичной работоспособности на двух фазах до уровня 66 \% от номинального, на одной фазе до $33 \%$ с дальнейшей остановкой электродвигателя.

Алгоритмы формирования мгновенного задания на фазные токи $I_{n}$ приведены в табл. 1 .

Параметр $k_{d}$ управления обеспечивает скачкообразное увеличение амплитуды фазных токов в аварийном режиме: $k_{d}=\left(d+\bar{d} \frac{3}{2}\right)$, для компенсации активной мощности отказавшей фазы двигателя: $d=1$ (отсутствие отказа) $k_{d}=1 ; \bar{d}=1$ (наличие отказа) $k_{d}=3 / 2, \theta_{\text {к }}$ угол перекрытия фаз двигателя $\left(\theta_{\mathrm{K}}=\pi / 4\right)[22]$.

\section{Результаты моделирования}

ВИД для ЭЦН в настоящее время серийно не выпускаются, поэтому для оценки мощности и напора ЭЦН необходимо подобрать известный аналог ЭЦН на основе асинхронного электродвигателя, получить на его основе требуемые параметры по

Таблица 1. Алгоритлы отказоустойчивого управления ВИД

Table 1. Fault-tolerant control algorithms of switched-reluctance motor

\begin{tabular}{|c|c|c|}
\hline № & $\begin{array}{l}\text { Наименование алгоритма управления } \\
\text { Control algorithm name }\end{array}$ & $\begin{array}{l}\text { Аналитическая запись алгоритма } \\
\text { Algorithm analytical form }\end{array}$ \\
\hline 0 & $\begin{array}{l}\text { Управление трехфазным вентильно-индукторным электроприводом с симметричной } \\
\text { oдиночной коммутацией и исходной отказоустойчивостью } \\
\text { Switched-reluctance drive control with symmetrical single switching and using initial } \\
\text { fault-tolerance }\end{array}$ & $\begin{array}{l}I_{A}=I_{\omega}\left[1+\operatorname{sign}\left(\varphi\left(i_{A}, \theta_{A}\right)-\theta\right)\right] / 2 \\
I_{B}=I_{\omega}\left[1+\operatorname{sign}\left(\varphi\left(i_{B}, \theta_{B}\right)-\theta\right)\right] / 2 \\
I_{C}=I_{\omega}\left[1+\operatorname{sign}\left(\varphi\left(i_{C}, \theta_{C}\right)-\theta\right)\right] / 2\end{array}$ \\
\hline 1 & $\begin{array}{l}\text { Алгоритм управления трехфазным вентильно-индукторным электроприводом с симме- } \\
\text { тричной одиночной коммутацией и компенсацией момента за счет увеличения ампли- } \\
\text { туды фазных токов } \\
\text { Switched-reluctance drive control algorithm with symmetrical single switching and torque } \\
\text { compensation account of phase current amplitude increasing }\end{array}$ & $\begin{array}{l}I_{A}=I_{\omega} \bar{a} k_{d}\left[1+\operatorname{sign}\left(\varphi\left(i_{A}, \theta_{A}\right)-\theta\right)\right] / 2 \\
I_{B}=I_{\omega} \bar{b} k_{d}\left[1+\operatorname{sign}\left(\varphi\left(i_{B}, \theta_{B}\right)-\theta\right)\right] / 2 \\
I_{C}=I_{\omega} \bar{c} k_{d}\left[1+\operatorname{sign}\left(\varphi\left(i_{C}, \theta_{C}\right)-\theta\right)\right] / 2\end{array}$ \\
\hline 2 & $\begin{array}{l}\text { Алгоритм управления трехфазным вентильно-индукторным электроприводом с ком- } \\
\text { пенсацией момента за счет изменения угла перекрытия фаз } \\
\text { Switched-reluctance drive control algorithm with torque compensation account of phase } \\
\text { overlap angle changing }\end{array}$ & $\begin{array}{l}I_{A}=I_{\omega} \bar{a}\left[1+\operatorname{sign}\left(\varphi\left(i_{A}, \theta_{A}\right)-\theta+d \theta_{\mathrm{K}}\right)\right] / 2 \\
I_{B}=I_{\omega} \bar{b}\left[1+\operatorname{sign}\left(\varphi\left(i_{B}, \theta_{B}\right)-\theta\right)+d \theta_{K}\right] / 2 \\
I_{C}=I_{\omega} \bar{c}\left[1+\operatorname{sign}\left(\varphi\left(i_{C}, \theta_{C}\right)-\theta\right)+d \theta_{K}\right] / 2\end{array}$ \\
\hline 3 & $\begin{array}{l}\text { Алгоритм управления трехфазным вентильно-индукторным электроприводом с ком- } \\
\text { пенсацией момента за счет изменения угла перекрытия фаз и увеличения амплитуд } \\
\text { фазных токов } \\
\text { Switched-reluctance drive control algorithm with torque compensation account of phase } \\
\text { overlap angle changing and phase current amplitude increasing }\end{array}$ & $\begin{array}{l}I_{A}=I_{\omega} \bar{a} k_{d}\left[1+\operatorname{sign}\left(\varphi\left(i_{A}, \theta_{A}\right)-\theta+d \theta_{K}\right)\right] / 2 ; \\
I_{B}=I_{\omega} \bar{b} k_{d}\left[1+\operatorname{sign}\left(\varphi\left(i_{B}, \theta_{B}\right)-\theta\right)+d \theta_{K}\right] / 2 ; \\
I_{C}=I_{\omega} \bar{c} k_{d}\left[1+\operatorname{sign}\left(\varphi\left(i_{C}, \theta_{C}\right)-\theta\right)+d \theta_{K}\right] / 2\end{array}$ \\
\hline
\end{tabular}


мощности и напору насосного агрегата. В качестве исходных параметров для расчёта приняты параметры реальной нефтяной скважины из [45]. С помощью MathCad рассчитаны параметры для моделирования: $H_{\mathrm{B}}=2214 \mathrm{M}, Q_{\mathrm{B}}=117,647 \quad \mathrm{~m}^{3} /$ сут, $P_{\text {гид }}=56,966$ кВт, где $H_{\text {в }}$ - необходимый напор насоса при работе на воде; $Q_{\text {в }}$ объемный расход насоса при работе на воде; $P_{\text {гид }}$ - гидравлическая мощность требуемого электродвигателя.

Из каталогов выбран ближайший подходящий по мощности и насосным характеристикам насос с асинхронным двигателем «ЭЦНа (К)5а-125(Тв)», который имеет характеристики: напор насоса $H_{\mathrm{B}}=2500 \mathrm{м}$, подача $Q_{\mathrm{B}}=125 \mathrm{~m}^{3} /$ сут, мощность $P=62,01$ кВт. Поэтому при моделировании ВИД принята мощность двигателя 60 кВт, получены характеристики напора и подачи близкие к аналогу [22], [24]. Параметры моделируемого ВИД приведены в табл. 2.

Таблица 2. Параметры моделируемого ВИд

Table 2. $\quad$ Simulating switched-reluctance motor parameters

\begin{tabular}{|c|c|}
\hline $\begin{array}{l}\text { Параметр } \\
\text { Parameter }\end{array}$ & $\begin{array}{c}\text { Величина } \\
\text { Value }\end{array}$ \\
\hline Сопротивление обмотки статора, 0м/Stator resistance, Ohm & 0,05 \\
\hline Момент инерции, кг" ${ }^{2} /$ Inertia of the shaft, $\mathrm{kg}^{\cdot} \mathrm{m}^{2}$ & 0,05 \\
\hline $\begin{array}{l}\text { Частота вращения под номинальной нагрузкой, об/мин } \\
\text { Speed under rated load, rpm }\end{array}$ & 3000 \\
\hline Момент трения на валу, H·м`c/Friction torque, $\mathrm{N} \cdot \mathrm{m} \cdot \mathrm{s}$ & 0,02 \\
\hline $\begin{array}{l}\text { Индуктивность в рассогласованном положении, Гн } \\
\text { Inductance in unaligned position, Gn }\end{array}$ & $0,67 \cdot 10^{-3}$ \\
\hline $\begin{array}{l}\text { Индуктивность в согласованном положении, Гн } \\
\text { Inductance in aligned position, Gn }\end{array}$ & $23,6 \cdot 10^{-3}$ \\
\hline $\begin{array}{l}\text { Индуктивность насыщения согласованного положения, Гн } \\
\text { Saturation inductance in aligned position, Gn }\end{array}$ & $0,15 \cdot 10^{-3}$ \\
\hline Максимальный ток, A/Maximum current, A & 450 \\
\hline $\begin{array}{l}\text { Максимальное потокосцепление, B·c } \\
\text { Maximum flux-linkage, V·s }\end{array}$ & 0,486 \\
\hline Напряжение питания, B/Supply voltage, V & 230 \\
\hline
\end{tabular}

При моделировании использованы параметры силового кабеля типа КПБП длинною до 1000 м. Такой кабель имеет токопроводящую шину диаметром 4,5 мм и сечение 16 мм² $^{2}$ В результате моделирования получено, что искажения питающего напряжения под влиянием КЛ не превышают $10 \%$ от номинального напряжения питания.

Рассмотрим напорно-расходные характеристики нефтедобывающего насоса с ВИД. На рис. 4, a приведена напорно-расходная характеристика для работы без алгоритмов: (строка 0 табл. 1), на рис. 4, б напорно-расходная характеристика при работе с применением алгоритма компенсации токов фаз (строка 1 табл. 1). Как следует из рис. 4, б, при применении алгоритма компенсации фазных токов возможно полное восстановление работоспособности на двух фазах.

На рис. 5 приведены характеристики объемного расхода жидкости при изменении напора $Q(H)$ и характеристики степенной зависимости момента от скорости с учетом времени $\left(\frac{\omega}{\omega_{\text {н }}}\right)^{x}(t)$, где $x$-сте-

пень зависимости (для кривых $1,4 x=4$, для кривых 2,5 $x=3$, для кривых $3,6 x=2$ ), при работе с применением алгоритма компенсации токов фаз (строка 1 табл. 1). Кривые 1-3 - характеристики степенной зависимости момента от скорости с учетом времени при различных $x$. Кривые 4-6 - зависимости объемного расхода жидкости от напора $Q(H)$ при разных $x$. До момента времени 0,5 ЭЦН работает в нормальном трехфазном режиме, далее с 0,5 по 1 в двухфазном режиме с алгоритмом компенсации тока при отказе одной фазы. При этом зависимости 4-6 напора $(H)$ от объемного расхода $(Q)$ пропорционально возрастают до уровня $Q=1$; $H=2500$ м, что соответствует номинальному режиму работы. С момента 1 до 1,5 с ЭЦН работает в однофазном режиме и характеристики 4-6 имеют спадающий характер до уровня $Q=0,55$; $\mathrm{H}=1200$ м. Из рисунка следует, что чем выше степенная зависимость, тем эффективнее алгоритм, то есть чем выше напор на магистрали, тем эффективнее восстановление производительности при активации алгоритма отказоустойчивого управления.

На рис. 6 представлены расходные характеристики объемного расхода жидкости для алгоритмов 0-3 из табл. 1 при последовательном аварийном отключении фаз электродвигателя. Численные значения установившегося уровня $Q$ при последовательном аварийном отключении фаз электродвигателя сведены в табл. 3.

Таблица 3. Значения объемного расхода жидкости при последовательном отключении фаз

Table 3. $\quad$ Fluid flow values trough the pump for sequential phase disconnection

Значения $Q$ для алгоритмов 0-3, в о.е 1 Фазы/Phases Fluid flow value $Q$ for algorithms $0-3$ in relative units \begin{tabular}{l|l|l|l|}
\cline { 2 - 5 } & 3 & 2 & 1
\end{tabular}

\begin{tabular}{|c|c|c|c|}
\hline$(0)$ & & 0,66 & 0,33 \\
\hline$(1)$ & 1 & 0,66 \\
\hline$(2)$ & & 0,9 & 0,59 \\
\hline$(3)$ & & 0,94 & 0,74 \\
\hline
\end{tabular}

Из анализа табл. 3 следует, что при использовании исходной отказоустойчивости (алгоритм 0) при неисправности одной фазы электродвигателя, установившееся значение объемного расхода жидкости составляет $66 \%$ от номинального уровня, при обрыве двух фаз электродвигателя - 33 \% соответственно. При этом показано, что двигатель может продолжать функционировать на одной оставшейся в работе фазе электродвигателя, что делает его уникальным по отношению к имеющимся в эксплуатации насосным агрегатам, построенным на основе асинхронного и вентильного двигателей [46, 47].

При работе с алгоритмом компенсации момента за счет увеличения амплитуды фазных токов (1) при неисправности одной фазы электродвигателя возможно полное восстановление работоспособно- 


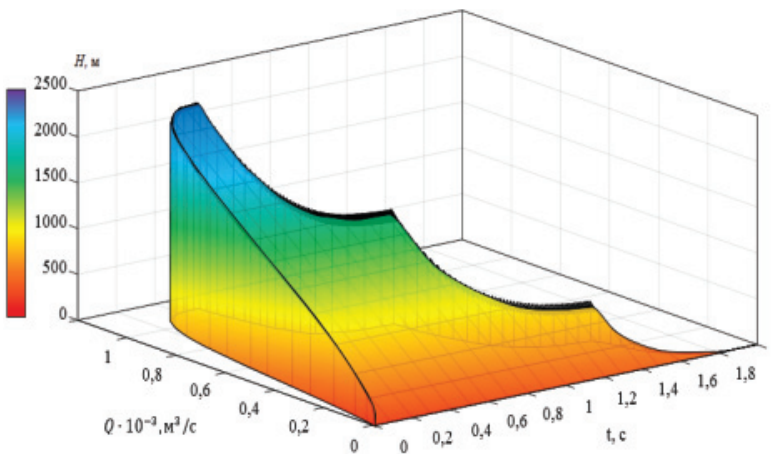

$a / a$

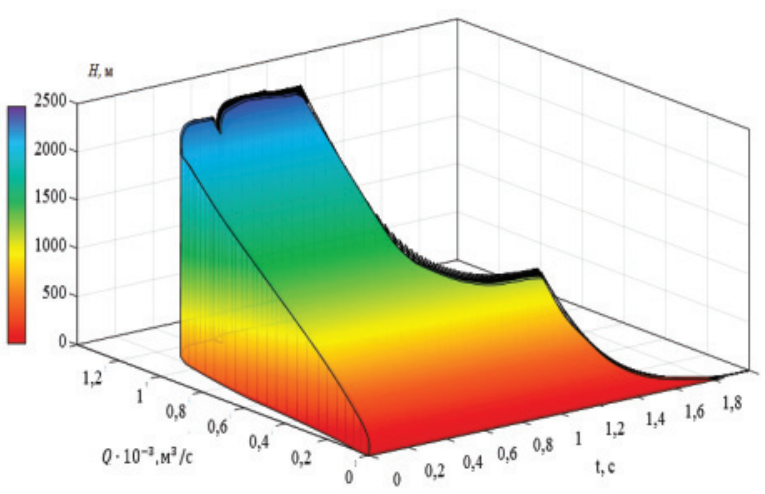

$\sigma / b$

Pис. 4. Напорно-расходная характеристика: а) работа без алгоритлов; б) работа с применением алгоритма компенсации молента за счет увеличения алплитуд токов фаз

Fig. 4. Pressure-flow characteristic: a) without algorithms; $b$ ) with torque compensation account of phase current amplitude increasing

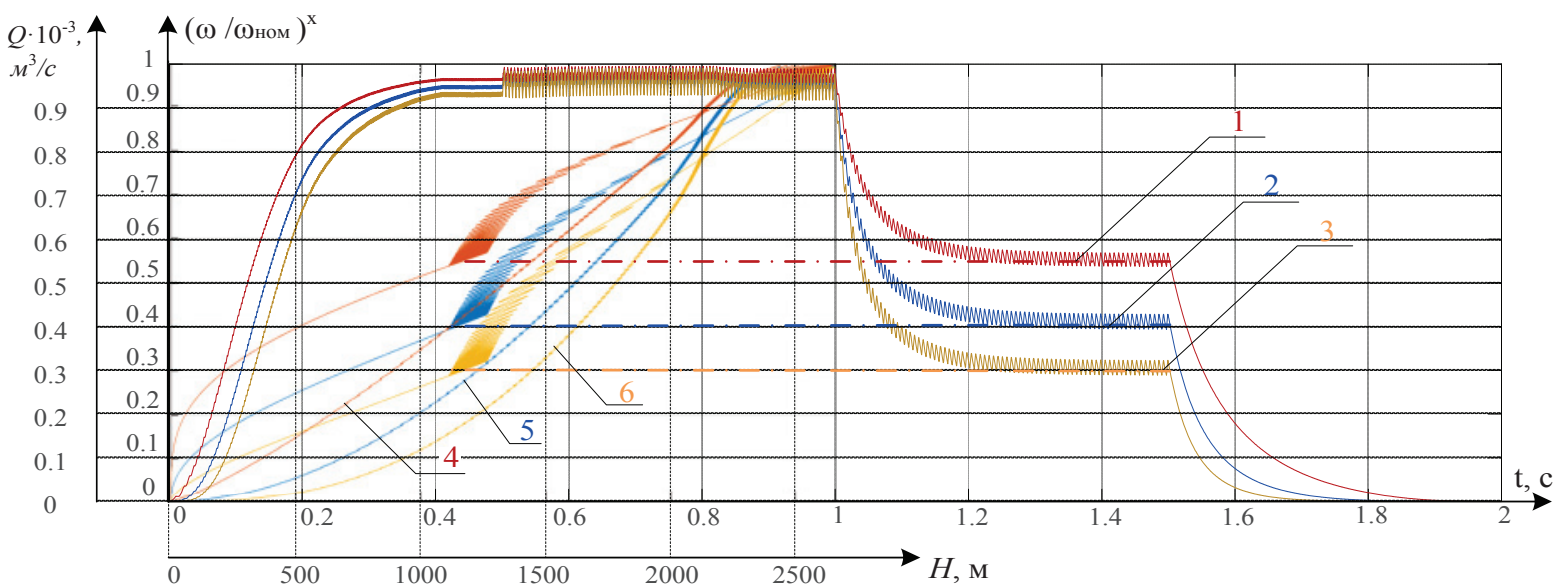

Puc. 5. Характеристики $Q(H)$ и характеристики степенной зависимости момента от скорости с учетом времени

Fig. 5. Characteristics $Q(H)$ and characteristics of the moment power-law dependence on speed during the time

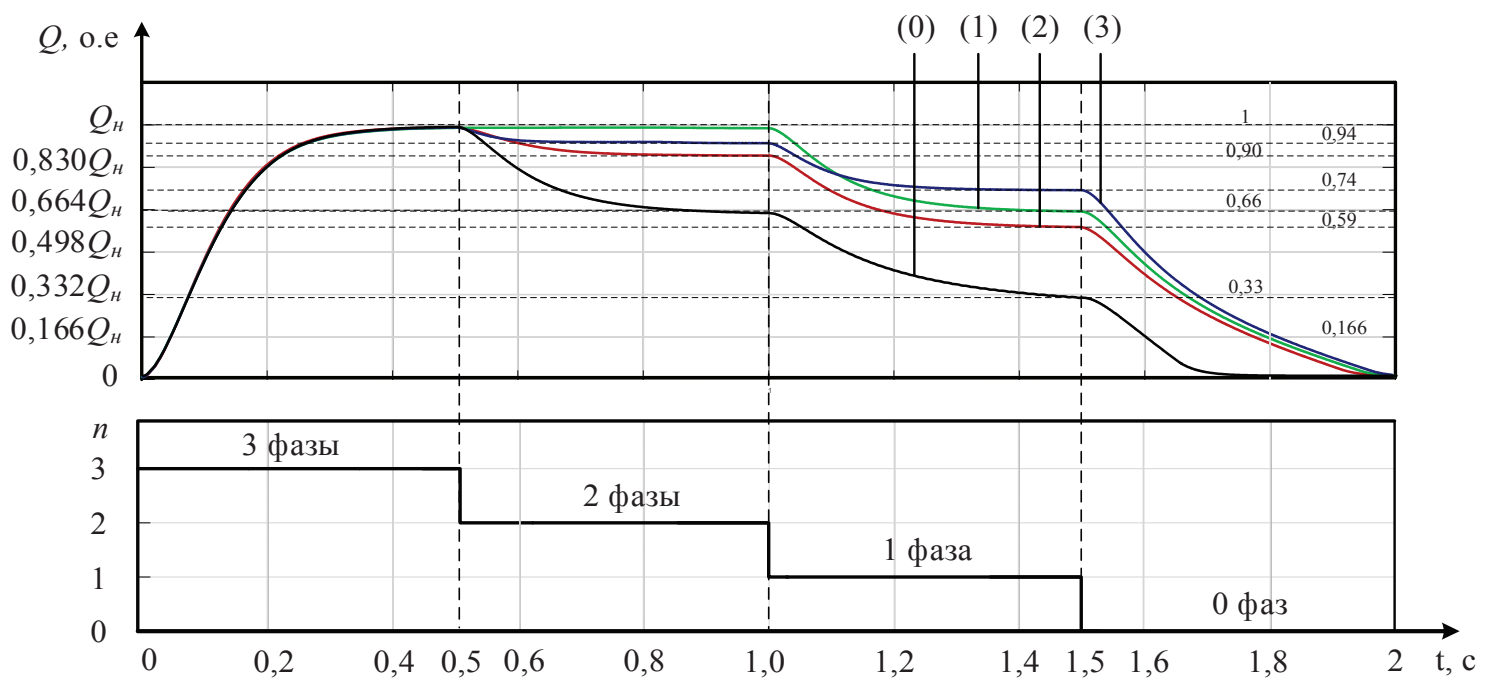

Pис. 6. Характеристики объемного расхода жидкости для алгоритмов 0-3 табл. 1

Fig. 6. Flow characteristic for operation with algorithms 0-3 from table 1 
сти без снижения заданного уровня объемного расхода жидкости. Данный алгоритм целесообразно применять, если требуемый уровень объемного расхода жидкости меньше номинального для электродвигателя, чтобы обеспечить долговременную эксплуатацию насоса при отказе электродвигателя типа «обрыв фазы» без риска перегрева обмоток электродвигателя. При неисправности типа «обрыв двух фаз» алгоритм 1 показал значение установившего уровня объемного расхода жидкости $66 \%$.

При работе с алгоритмом 2 с компенсацией момента за счет изменения угла перекрытия фаз установившиеся значения составили при неисправности типа «обрыв фазы» электродвигателя 90 \% и при неисправности типа «обрыв двух фаз» электродвигателя $59 \%$ соответственно. Алгоритм 2 позволяет без каких-либо конструктивных изменений и риска перегрева повысить отказоустойчивость исполнительного электродвигателя, однако при этом возрастает потребление мощности от источника питания [26].

При работе комбинированного алгоритма 3 с компенсацией момента за счет изменения угла перекрытия фаз и увеличения амплитуд фазных токов в 1,5 раза при неисправности типа «обрыв одной фазы» установившееся значение объемного расхода жидкости составило $94 \%$, а при неисправности типа «обрыв двух фаз» - 74 \% .

Предложенные алгоритмы отказоустойчивого управления для погружных электроцентробежных насосов применимы для управления ВИД с наблюдателем состояния или с датчиком положения ротора. Применительно к данной статье, использовать предложенные алгоритмы отказоустойчивого управления электроцентробежными насосными установками с ВИД в настоящее время возможно только для их наземного исполнения, однако при дальнейших доработках с учетом бездатчикового управления возможно использование ВИД в качестве погружного электродвигателя насосного агрегата добычи нефти.

\section{Выводы}

1. Разработаны математическая и имитационная модели отказоустойчивого трехфазного вентильно-индукторного электропривода насосного агрегата. Исходя из анализа напорно-расходных характеристик следует, что применение алгоритмов отказоустойчивого управления по-

\section{СПИСОК ЛИТЕРАТУРЫ}

1. Российский рынок нефтесервисных услуг 2014. Аналитический обзор / под ред. Е.М. Миролюбовой. - М.: РБК, 2014. $218 \mathrm{c.}$

2. Ивановский В.Н. Анализ современного состояния и перспектив развития скважных насосных установок для добычи нефти // Территория Нефтегаз. - 2007. - № 11. - С. 36-47.

3. Стекольников Ю.И. Живучесть систем. - СПб.: Политехника, 2002. $-155 \mathrm{c}$. зволяет повысить отказоустойчивость исполнительного трехфазного ВИД без конструктивных изменений.

2. Показано, что при использовании исходной отказоустойчивости с неисправностью в одной фазе электродвигателя установившееся значение потока жидкости составляет 66 \% от номинального уровня, при неисправности типа «обрыв двух фаз» электродвигателя - 33 \% соответственно. При этом показано, что электродвигатель может продолжать функционировать на одной оставшейся в работе фазе, что делает его уникальным по отношению к имеющимся в эксплуатации насосам, построенным на основе асинхронного и вентильного двигателей.

3. При работе с алгоритмом компенсации момента за счет увеличения амплитуды фазных токов при неисправности одной фазы электродвигателя возможно полное восстановление работоспособности без снижения заданного уровня объемного расхода жидкости. Данный алгоритм целесообразно применять, если требуемый уровень объемного расхода жидкости меньше номинального для электроцентробежного насоса, чтобы обеспечить долговременную эксплуатацию насосного агрегата при отказе электродвигателя типа «обрыв фазы» без риска перегрева обмоток электродвигателя. При неисправности типа «обрыв двух фаз» этот алгоритм показал значение установившего уровня объемного расхода жидкости $66 \%$.

4. При работе с алгоритмом с компенсацией момента за счет изменения угла перекрытия фаз установившиеся значения составили при неисправности типа «обрыв фазы» электродвигателя 90 \% и при неисправности «обрыв двух фаз» электродвигателя - 59 \% соответственно. Алгоритм позволяет без каких-либо конструктивных изменений и риска перегрева повысить отказоустойчивость исполнительного электродвигателя, однако при этом возрастает потребление мощности от источника питания.

5. При работе комбинированного алгоритма с компенсацией момента за счет изменения угла перекрытия фаз и увеличения амплитуд фазных токов в 1,5 раза при неисправности типа «обрыв фазы» установившееся значение потока жидкости составило 94 \%, а при неисправности типа «обрыв двух фаз» - $74 \%$.

4. Боловин Е.В., Глазырин А.С. Метод идентификации параметров погружных асинхронных электродвигателей установок электроприводных центробежжых насосов для добычи нефти // Известия Томского политехнического университета. Инжиниринг георесурсов. - 2017. - Т. 328. - № 1. - С. 123-131.

5. Однокопылов Г.И., Саркисов Д.Ю. Оценка параметров разрушающей нагрузки при ударно-волновом нагружении для ответственных строительных конструкций сооружений нефтегазового комплекса // Известия Томского политехнического 
университета. Инжиниринг георесурсов. - 2017. - Т. 328. № 3. - С. $85-95$.

6. Однокопылов Г.И., Шевчук В.А., Дементьев Ю.Н. Применение системного анализа для обеспечения эксплуатационной надёжности электрических машин в алмазодобывающей промышленности // Известия Томского политехнического университета. Инжиниринг георесурсов. - 2019. - Т. 330. - № 5. C. $131-140$.

7. Нгуен Куанг Кхоа. Исследование электромеханического комплекса: вентильно-индукторный электропривод - центробежный насос // Известия высших учебных заведений. Электромеханика. - 2016. - № 4. - С. 55-64.

8. Вентильно-индукторный электропривод для насоса ГРАТ1800/67 / Г.К. Птах, А.А. Цветков, И.А. Квятковский, Д.А. Протасов, Д.В. Рожков // Известия Тульского Государственного университета. Технические науки. - 2010. - № 3. C. $227-231$.

9. Ясаков Г.С., Агафонов В.В., Костиков Е.А. Погружной вентильно-индукторный электродвигатель открытого исполнения с универсальными подшипниками скольжения // Вестник Севастопольского национального технического университета. 2012. - № 132. - C. 50-53.

10. Odnokopylov G.I., Bragin A.D. Fault tolerant vector control of induction motor drive (Article number 012015) // IOP Conference Series: Materials Science and Engineering. - 2014. - V. 66. № 1. - P. 1-6.

11. Сравнительный анализ отказоустойчивого управления асинхронного и вентильно-индукторного электропривода / Г.И. Однокопылов, И.А. Розаев, А.Д. Брагин, Е.П. Сенькив // Интеллектуальные энергосистемы: труды IV Международного молодёжного форума. - Томск, 2016. - С. 89-93.

12. Odnokopylov G.I., Bragin A.D. Mathematical model of brushless DC motor in phase loss operation mode // Applied Mechanics and Materials. - 2015. - V. 698. - P. 24-29.

13. Odnokopylov G.I., Bragin A.D. Algorithms of fault tolerant control of induction motor electric drive in phase loss operate mode // 2015 International Siberian Conference on Control and Communications (SIBCON): Proc. - Omsk, May 21-23, 2015. - P. 1-5.

14. Птах Г.К. Вентильно-индукторный реактивный электропривод средней и большой мощности: зарубежный и отечественный опыт // Электротехника: Сетевой электронный научный журнал. - 2015. - Т. 2. - № 3. - С. 23-33.

15. Вигриянов П.Г. Энергетические характеристики многофазного вентильного двигателя в нормальных и аварийных режимах // Вентильные электромеханические системы с постоянными магнитами: Всесоюзная научно-техническая конференция. Тез. Докл. - М., 1989. - С. 14.

16. Кузнецов В.А., Кузьмичев В.А. Вентильно-индукторные двигатели. - М.: Изд-во МЭИ, 2003. - 70 с.

17. Алямкин Д.И. Разработка и исследование двухфазного вентильно-индукторного электропривода насосов горячего водоснабжения: дис. ... канд. техн. наук. - М., 2011. - 229 с.

18. About the using of capacitive energy storages in the frequencycontrolled electric drives / I. Braslavsky, Z. Ishmatov, Y. Plotnikov, G. Erman // Proc. of Symposium on Power Electronics, Electric Drives, Automation and Motion. - Taormina, Italy, 2012. P. $920-925$

19. Ishmatov Z., Plotnikov I., Braslavsky I. About application perspective of capacitive energy storage in the frequency-controlled electric drive $/ / 9^{\text {th }}$ International Conference on Power Drives Systems (ICPDS 2016). - Perm, 2016. - P. 123-127.

20. Plotnikov I., Polyakov V., Postnikov N. Single-Loop Control System for Energy Storage Device in the Frequency-Controlled Electric Drive / / X International Conference on Electrical Power Drive Systems (ICEPDS). - Ekaterinburg, 2018. - P. 1-5.

21. Polyakov V., Plotnikov I., Postnikov N. Three-Loop Control System of Energy Storage Device in the Frequency-Controlled Elec- tric Drive $/ / 26^{\text {th }}$ International Workshop on Electric Drives: Improvement in Efficiency of Electric Drives (IWED). - Moscow, 2019. - P. 1-5.

22. Однокопылов Г.И. Методы и алгоритмы отказоустойчивого управления электроприводами опасных производственных объектов: дис. ... д-ра техн. наук. - Томск, 2017. - 343 с.

23. Krishnan R., Lim H.S. Novel measurement disturbance rejection current control for linear switched reluctance motor drives // Conference record - IAS annual meeting (IEEE industry applications society). - Blacksburg, VA: Virginia Tech, 2007. P. 2226-2233.

24. Odnokopylov G.I., Rozayev I.A. Formation of failure matrix and failure-free control algorithm for multi-sectioned Switched-reluctance drive (Article number 012035) // IOP Conference Series: Materials Science and Engineering. - 2014. - V. 66. - № 1. - P. 1-7.

25. Odnokopylov G.I., Rozaev I.A. Fault-tolerant control algorithms of switched-reluctance motor drive in open-phase modes $/ / 11^{\text {th }}$ International Forum on Strategic Technology (IFOST): Proc. Novosibirsk, June 1-3, 2016. - Novosibirsk: Novosibirsk State Technical University, 2016. - V. 2. - P. 140-144.

26. Popov M.M., Maniv 0. Simulation of an autonomous power supply system based on lithium-iron-phosphate (LIFEP04) // Smart Grids 2017: MATEC web of conferences: $5^{\text {th }}$ International Youth Forum: proceedings. - Tomsk, 2017. - P. 01060. DOI: 10.1051/matecconf/201714101060

27. Шевчук В.А., Муравлев 0.П. Анализ вероятности безотказной работы электрических машин в алмазодобывающей промышленности // Горное оборудование и электромеханика. 2018. - № 4 (138). - C. 39-46.

28. Ткачук Р.Ю., Глазырин А.С. Принцип построения отказоустойчивой системы управления асинхронным электроприводом // Известия Томского политехнического университета. 2012. - T. 321. - № 5. - C. 105-109.

29. Нугаев И.Ф., Искужин Р.В. Комплекс математических моделей для решения задач синтеза алгоритмов управления процессами в нефтедобывающих скважинах // Вестник Уфимского государственного авиационного технического университета. - 2012. - № 7. - С. 36-44.

30. Александровский С.В., Петренко Ю.Н. Разработка математической и имитационной модели вентильно-индукторного двигателя // Энергетика. Известия высших учебных заведений и энергетических объъединений СНГ. - 2011. - № 2. - С. 15-22.

31. Investigating Measurement Errors in Dual-Frequency Probing Technique by Mathematical Modeling / Y.V. Shul'gina, M.A. Kostina, A.I. Soldatov, A.A. Soldatov, P.V. Sorokin // Russian Journal of Nondestructive Testing. - 2019. - V. 55. - № 1. P. 15-21.

32. Miller T.J. Switched reluctance motors end their control. - Virginia: Oxford Magna Physics Publishing and Clarendon Press, 1993. $-205 \mathrm{p}$.

33. Ruba M., Anders M. Fault Tolerant Switched Reluctance Machine Study // Proc. of the International Conference on Power Electronics, Intelligent Motion and Power Quality (PCIM 2008). Nürnberg, Germany, 2008. -6 p.

34. Темирёв А.П. Математическое моделирование, проектирование и экспериментальное определение параметров вентильноиндукторных электроприводов: монография. - Новочеркасск: ЛИК, 2011. - 794 с.

35. Нугаев И.Ф., Искужин Р.В. Динамическая модель нефтедобывающей скважины на базе УЭцН как объекта управления // Нефтегазовое дело. - 2012. - № 5. - С. 31-46.

36. Нугаев И.Ф., Искужин Р.В. Комплекс математических моделей для синтеза алгоритмов управления процессами в нефтедобывающих скважинах // Вестник УГАТУ. - 2012. - Т. 16. № 8 (53). - C. 36-44.

37. Gerling D., Schramm A. Evaluation and Comparison of Fault Tolerant Switched Reluctance Machines for a Specific Application: 
Proc. of the 9 Spanish-Portuguese Congress on Electrical Engineering (9CHLIE). - Marbella, Spain, - 2005. - 5 p.

38. Нгуен Куанг Кхоа. Методика моделирования вентильно-индукторных двигателей с помощью программ Elcut и Matlab Simulink // Изв. вузов. Электромеханика. - 2016. - № 2. C. $73-79$.

39. Черных И.В. Моделирование электротехнических устройств в MATLAB, SimPowerSystems и Simulink. - М.: ДМК Пресс; СПб: Питер, 2008. - 288 с.

40. Любарский Б.Г., Рябов Е.С. Моделирование электроприводов на основе реактивных индукторных двигателей в среде MatLab Simulink // Проектирование инженерных и научных приложений в среде MATLAB: труды V международной научной конференции. - Харьков, 11-13.05.2011 - Харьков: Изд-во Национального технического университета «Харьковский политехнический институт», 2011. - С. 404-424. URL: https://matlab.ru/upload/resources/EDU\% 20Conf/pp\% 20404-424\% 20Ljubarskiy.pdf (дата обращения 25.09.2019).

41. Study of a 3 phase $(6 / 4)$ switched reluctance motor control / N.D. Irimia, A. Simon, L. Livadaru, S. Vlasceanu, 0. Dabija, A.M. Mihai // Buletinul AGIR. - Iasi, Romania, 2011. P. $129-134$

42. Павлов Д.О., Кузнецов Е.М. Бездатчиковое определение скорости в электроприводе установок электроцентробежных насосов // Актуальные вопросы энергетики. - Омск: Изд-во Омского государственного технического университета, 2016. - С. 147-153.
43. Чавылалов М.В. Бездатчиковое определение положения ротора в системе управления вентильно-индукторного электропривода: дис. ... канд. техн. наук. - Ростов-на-Дону, 2013. $115 \mathrm{c.}$

44. Результаты опытно-промышленных испытаний энергоэффективных УЭЦН ЗАО «Новомет-Пермь» / М.С. Попов, Е.Г. Ветохин, М.Н. Каверин, В.П. Тарасов // Оборудование и технологии для нефтегазового комплекса. - 2012. - № 3. - С. 63-74.

45. Мордвинов В.А., Турбаков М.С. К методике выбора электроцентробежных насосов при эксплуатации нефтедобывающих скважин // Вестник Пермского государственного технического университета. Геология, геоинформационные системы, горно-нефтяное дело. - 2009. - Т. 8. - № 4. - С. 59-67.

46. Алгоритмы управления и обеспечение устойчивости системы резервного питания частотно-регулируемого электропривода от сети постоянного тока / В.В. Вдовин, Д.П. Вислогусов, В.А. Клан, Д.А. Котин, В.В. Панкратов, А.В. Сметанников // Электротехника. - 2015. - Т. 86. - № 8. - С. 54-58.

47. Нос 0.В., Волков В.Ю., Клан В.А. Векторное управление электроприводом переменного тока с многоуровневым преобразователем частоты на базе н-мостов / Электротехника. - 2019. T. 90. - № 4. - C. 13-19.

Поступила 30.09.2019 г.

\section{Информация об авторах}

Однокопылов Г.И., доктор технических наук, профессор отделения электроэнергетики и электротехники Инженерной школы энергетики, Национальный исследовательский Томский политехнический университет.

Букреев В.Г., доктор технических наук, профессор, профессор отделения электроэнергетики и электротехники Инженерной школы энергетики, Национальный исследовательский Томский политехнический университет.

Розаев И.А., ассистент отделения электроэнергетики и электротехники Инженерной школы энергетики, Национальный исследовательский Томский политехнический университет. 


\title{
RESEARCH OF FAULT-TOLERANT SWITCHED-RELUCTANCE MOTOR OF ELECTRICAL OIL PUMP
}

\author{
Georgy I. Odnokopylov', \\ OGIz@yandex.ru
}

\author{
Viktor G. Bukreev', \\ Bukreev@tpu.ru \\ Ivan A. Rozaev', \\ Veazor@tpu.ru \\ ${ }^{1}$ National Research Tomsk Polytechnic University,
30, Lenin Avenue, Tomsk, 634050, Russia.
}

The relevance. Nowadays oil production is one of the priority areas of industrial development in the country. It leads to requiring further research related to increasing the efficiency of the electric motor included in the installation of the centrifugal pump. The emergency failure of an electrical motor entails to well shutdown, which leads to significant economic losses. Therefore, ensuring the fault tolerance of the executive motor is one of the most important tasks for oil and gas complex. To eliminate well shutdown and ensure production continuity in the event of a motor phase failure, it is necessary to use a promising type of electromechanical energy converter such as a switched-reluctance motor. It is crucial issue to develop and improve control systems and implement fault-tolerant control algorithms witch currently have not found application in oil production.

The main aim of the research is to investigate the operation possibilities of the switched-reluctance electric motor in emergency nonphase operation modes; to develop fault-tolerant control algorithms to preserve and restore the operability of the switched-reluctance motor with single and multiple failures of power circuits.

Object of the research is three-phase switched-reluctance motor of pump in non-phase operation modes, operated in oil production. Methods: mathematical theory of electric machines; numerical methods used in description of dynamic electrical, mechanical and magnetic processes; mathematical modeling and programming in MathCad and Matlab Simulink.

Results. The authors have developed the mathematical and simulation model of a fault-tolerant three-phase switched-reluctance drive, obtained and analyzed the pressure-flow characteristics of operation in an emergency non-phase mode using algorithms of fault-tolerant control of a switched-reluctance motor.

\section{Key words:}

Switched-reluctance motor, phase failure, fault-tolerant electric drive, fault-tolerant control algorithm, pressure-flow pump characteristics.

\section{REFERENCES}

1. Rossiyskiy rynok nefteservisnykh uslug 2014. Analitichesky obzor [Russian oil and gas services market 2014. Analytical Review]. Ed. by E. Mirolyubova. Moscow, RBK Publ., 2014. 218 p.

2. Ivanovskiy V.N. Analiz sovremennogo sostoyaniya i perspektiv razvitiya skvazhnykh nasosnykh ustanovok dlya dobychi nefti [Analysis of the current state and development prospects of downhole pumping units for oil production]. Neftegaz Territory, 2007, Iss. 11. pp. 36-47.

3. Stekolnikov Yu.I. Zhivuchest system [System survivability]. StPetersburg, Politekhnika Publ., 2002. 155 p

4. Bolovin E.V., Glazyrin A.S. Method for identifying parameters of submersible induction motors of electrical submersible pump units for oil production. Bulletin of the Tomsk Polytechnic University. Geo Assets Engineering, 2017, vol. 328, Iss. 1, pp. 123-131. In Rus.

5. Odnokopylov G.I., Sarkisov D.Yu. Evaluation of breaking load parameters under shock wave loading for critical constructions of oil and gas sector facilities. Bulletin of the Tomsk Polytechnic University. Geo Assets Engineering, 2017, vol. 328, no. 3, pp. 85-95. In Rus.

6. Odnokopylov G.I., Shevchuk V.A., Dementyev Yu.N. Application of system analysis for providing reliability of electrical machines in diamond industry. Bulletin of the Tomsk Polytechnic University. Geo Assets Engineering, 2019, vol. 330, no. 5, pp. 131-140. In Rus.

7. Nguyen Kuang Kkhoa. Issledovanie elektromekhanicheskogo kompleksa: ventilno-induktorny elektroprivod - tsentrobezhny nasos [Research of electromechanical complex: switched reluctance drive - centrifugal pump]. Letters of higher educational institutions. Electromechanics, 2016, no. 4, pp. 55-64.

8. Ptakh G., Tsvetkov A., Kvyatkovsky I., Protasov D., Rogkov D. Ventilno-induktorny elektroprivod dlya nasosa GRAT-1800/67 [The switched-reluctance electric drive for the pump GRAT1800/67]. Notes of the Tula state university. Technical science, 2010, no. 3, pp. 227-231.

9. Yasakov G.S., Agafonov V.V., Kostikov E.A. Pogruzhnoy ventilno-induktorny elektrodvigatel otkrytogo ispolneniya s universalnymi podshipnikami skolzheniya [Submersible open type switch reluctance drive with universal sleeve type bearing]. Bulletin of Sevastopol National Technical University, 2012, no. 132, pp. 50-53.

10. Odnokopylov G.I., Bragin A.D. Fault tolerant vector control of induction motor drive (Article number 012015). IOP Conference Series: Materials Science and Engineering, 2014, vol. 66, no. 1, pp. $1-6$.

11. Odnokopylov G.I., Rozaev I.A., Bragin A.D., Senkiv E.V. Sravnitelny analiz otkazoustoychivogo upravleniya asinkhronnogo i ventilno-induktornogo elektroprivoda [Comparative analysis of fault-tolerant control of an induction and switched-reluctance electric drive]. Intellektualnye energosistemy: trudy IV Mezhdunarodnogo molodyozhnogo foruma [Intelligent power systems: proceedings of the IV International Youth Forum]. Tomsk, 2016. pp. 89-93.

12. Odnokopylov G.I., Bragin A.D. Mathematical model of brushless DC motor in phase loss operation mode. Applied Mechanics and Materials, 2015, vol. 698, pp. 24-29. 
13. Odnokopylov G.I., Bragin A.D. Algorithms of fault tolerant control of induction motor electric drive in phase loss operate mode. 2015 International Siberian Conference on Control and Communications (SIBCON): proceedings. Omsk, May 21-23, 2015. Novosibirsk, 2015. pp. 1-5.

14. Ptah G.K. Ventilno-induktorny reaktivny elektroprivod sredney i bolshoy moshchnosti: zarubezhny i otechestvenny opyt [Switched Reluctance Drive Medium and High Power: Foreign and Domestic Experience]. Electrical equipment: network electronic scientific magazine, 2015, vol. 2, no. 3, pp. 23-33.

15. Vigriyanov P.G. Energeticheskie kharakteristiki mnogofaznogo ventilnogo dvigatelya $\mathrm{v}$ normalnykh $\mathrm{i}$ avariynykh rezhimakh [Energy characteristics of a multiphase valve motor in normal and emergency conditions]. Ventilnye elektromekhanicheskie sistemy s postoyannymi magnitami. Vsesoyuznaya nauchnotekhnicheskaya konferetsiya. Tezisy dokladov [All-Union Scientific and Technical Conference. Valve Electromechanical Systems with Permanent Magnets: report thesis]. Moscow, 1989. pp. 14.

16. Kuznetsov V.A., Kuzmichev V.A. Ventilno-induktornye dvigateli [Switched-reluctance motors]. Moscow, MEI Publ., 2003. 70 p.

17. Alyamkin D.I. Razrabotka i issledovanie dvukhfaznogo ventilno-induktornogo elektroprivoda nasosov goryachego vodosnabzheniya. Dis. Kand. nauk [Development and research of a two-phase switched-reluctance electric drive of hot water pumps. Cand. Diss.]. Moscow, 2011. 229 p.

18. Braslavsky I., Ishmatov Z., Plotnikov Y., Erman G. About the using of capacitive energy storages in the frequency-controlled electric drives. Proceeding of Symposium on Power Electronics, Electric Drives, Automation and Motion. Taormina, Italy, 2012. pp. 920-925.

19. Ishmatov Z., Plotnikov I., Braslavsky I. About application perspective of capacitive energy storage in the frequency-controlled electric drive. $9^{\text {th }}$ International Conference on Power Drives Systems, ICPDS 2016. Perm, 2016. pp. 123-127.

20. Plotnikov I., Polyakov V., Postnikov N. Single-Loop Control System for Energy Storage Device in the Frequency-Controlled Electric Drive. 2018 X International Conference on Electrical Power Drive Systems (ICEPDS). Ekaterinburg, 2018. pp. 1-5.

21. Polyakov V., Plotnikov I., Postnikov N. Three-Loop Control System of Energy Storage Device in the Frequency-Controlled Electric Drive. $201926^{\text {th }}$ International Workshop on Electric Drives: Improvement in Efficiency of Electric Drives (IWED). Moscow, 2019. pp. 1-5.

22. Odnokopylov G.I. Metody i algoritmy otkazoustoychivogo upravleniya elektroprivodami opasnykh proizvodstvennykh obyektov. Dis. Dokt. nauk [Methods and algorithms of fault-tolerant control of electric drives of industrial production. Dr. Diss.]. Tomsk, 2017. $343 \mathrm{p}$.

23. Krishnan R., Lim H.S. Novel measurement disturbance rejection current control for linear switched reluctance motor drives. Con ference record - IAS annual meeting (IEEE industry applications society). Blacksburg, VA, Virginia Tech, 2007. pp. 2226-2233.

24. Odnokopylov G.I., Rozayev I.A. Formation of failure matrix and failure-free control algorithm for multi-sectioned Switched-reluctance drive (Article number 012035). IOP Conference Series: Materials Science and Engineering, 2014, vol. 66, no. 1, pp. 1-7.

25. Odnokopylov G.I., Rozaev I.A. Fault-tolerant control algorithms of switched-reluctance motor drive in open-phase modes. IFOST2016: $11^{\text {th }}$ International Forum on Strategic Technology. Novosibirsk, 1-3 June 2016. Novosibirsk, Novosibirsk State Technical University, 2016. Vol. 2, pp. 140-144.

26. Popov M.M., Maniv 0. Simulation of an autonomous power supply system based on lithium-iron-phosphate (LIFEP04). MATEC web of conferences: $5^{\text {th }}$ International Youth Forum. Smart Grids 2017: proceedings. Tomsk, October 9-13, 2017. pp. 01060. D0I: $10.1051 /$ matecconf $/ 201714101060$
27. Shevchuk V.A., Muravlev 0.P. Analysis of failure-free operation probability for electrical machines in the diamond industry. $\mathrm{Mi}$ ning equipment and electrical engineering, 2018, vol. 4, no. 138, pp. 39-46. In Rus.

28. Tkachuk R.Yu., Glazyrin A.S. The principle of control system building a fault-tolerant induction drive. Bulletin of the Tomsk Polytechnic University, 2012, vol. 321, no. 5. pp. 105-109. In Rus.

29. Nugayev I.F., Iskuzhin R.V. Kompleks matematicheskikh modeley dlya resheniya zadach sinteza algoritmov upravleniya protsessami v neftedobyvayushchikh skvazhinakh [A complex of mathematical models for solving the problems of synthesis of process control algorithms in oil wells]. Bulletin of the Ufa State Aviation Technical University, 2012, vol. 7, pp. 36-44.

30. Aleksandrovskiy S.V., Petrenko Yu.N. Razrabotka matematicheskoy i imitatsionnoy modeli ventilno-induktornogo dvigatelya [Development of mathematical and simulation models of switched-reluctance motor]. Energetika. Izvestiya vysshikh uchebnykh zavedeniy i energeticheskikh ob'edineniy SNG, 2011, vol. 2, pp. 15-22.

31. Shul'gina Y.V., Kostina M.A., Soldatov A.I., Soldatov A.A., Sorokin P.V. Investigating Measurement Errors in Dual-Frequency Probing Technique by Mathematical Modeling. Russian Journal of Nondestructive Testing, 2019, vol. 55, no. 1, pp. 15-21.

32. Miller T.J. Switched reluctance motors end their control. Virginia, Oxford Magna Physics Publishing and Clarendon Press, 1993. $205 \mathrm{p}$.

33. Ruba M., Anders M. Fault Tolerant Switched Reluctance Machine Study. Proceedings of the International Conference on Power Electronics. Intelligent Motion and Power Quality (PCIM '2008). Nurnberg, Germany, 2008. 6 p.

34. Temirev A.P. Matematicheskoe modelirovanie. Proyektirovanie $i$ eksperimentalnoe opredelenie parametrov ventilno-induktornykh elektroprivodou. Monografiya [Math modeling. Design and experimental determination of the parameters of switched-reluctance electric drives. Monograph]. Novocherkassk, LIK Publ., 2011. $794 \mathrm{p}$.

35. Nugayev I.F., Iskuzhin R.V. Dinamicheskaya model neftedobyvayushchey skvazhiny na baze UETsN kak obyekta upravleniya [A dynamic model of an oil well based on the EEC as a control object]. Neftegazovoe delo, 2012, vol. 5, pp. 31-46.

36. Nugayev I.F., Iskuzhin R.V. A complex of mathematical models for the synthesis of process control algorithms in oil wells. Bulletin of USATU, 2012, vol. 16, no. 8 (53), pp. 36-44. In Rus

37. Gerling D., Schramm A. Evaluation and Comparison of Fault Tolerant Switched Reluctance Machines for a Specific Application. Proc. of the $9^{\text {th }}$ Spanish-Portuguese Congress on Electrical Engineering (9CHLIE). Marbella, Spain, 2005, 5 p.

38. Nguyen Kuang Kkhoa. A method of simulation of the switched reluctance motors using programs Elcut and Matlab Simulink. University News. Electromechanics, 2016, no. 2, pp. 73-79.

39. Chernykh I.V. Modelirovanie elektrotekhnicheskikh ustroystv $v$ MATLAB, SimPowerSystems i Simulink [Modeling of electrical devices in MATLAB, SimPoverSystems and Simulink]. Moscow, DMK Press; St-Petersburg, Piter Publ., 2008. 288 p.

40. Lyubarskiy B.G., Ryabov E.S. Modelirovanie elektroprivodov na osnove reaktivnykh induktornykh dvigateley v srede MatLab Simulink [Modeling electric drives based on jet induction motors in MatLab Simulink environment]. Proektirovanie inzhenernykh $i$ nauchnykh prilozheny $v$ srede MATLAB. Trusy V Mezhdunarodnoy nauchnoy konferentsii [Design of engineering and scientific applications in MATLAB. Proc. of the V International scientific conrference]. Kharkov, 11-13 May 2011. Kharkov, National Technical University "Kharkov Polytechnic Institute», 2011. pp. 404-424. Available at: https://matlab.ru/upload/resources/EDU\% 20Conf/pp\% 20404-424 \% 20Ljubarskiy.pdf (accessed 25 September 2019). 
41. Irimia N.D., Simon A., Livadaru L., Vlasceanu S., Dabija 0., Mihai A.M. Study of a 3 phase $(6 / 4)$ switched reluctance motor control. Buletinul AGIR. Iasi, Romania, 2011. pp. 129-134.

42. Pavlov D.0., Kuznetsov E.M. Bezdatchikovoe opredelenie skorosti v elektroprivode ustanovok elektrotsentrobezhnykh nasosov [Sensorless speed detection in the electric drive of electric centrifugal pump installations]. Aktualnye voprosy energetiki [Actual issues of power engineering]. Omsk, Omsk State Technical University Publ., 2016. pp. 147-153.

43. Chavylalov M.V. Bezdatchikovoe opredelenie polozheniya rotora $v$ sisteme upravleniya ventilno-induktornogo elektroprivoda. Dokt. Dis. [Sensorless positioning of the rotor in the control system of a switched-reluctance electric drive. Dr. Diss.]. Rostov-on-Don, $2013.115 \mathrm{p}$.

44. Popov M.S., Vetokhin E.G., Kaverin M.N., Tarasov V.P. Rezultaty opytno-promyshlennykh ispytaniy energoeffektivnykh UECN ZA0 «Novomet-Perm» [Results of pilot tests of energy-efficient ESPs of «Novomet-Perm» CJSC]. Oborudovanie i tekhnologii dlya neftegazovogo kompleksa, 2012, no. 3, pp. 63-74.

\section{Information about the authors}

Georgy I. Odnokopylov, Dr. Sc., professor, National Research Tomsk Polytechnic University.

Victor G. Bukreev, Dr. Sc., professor, National Research Tomsk Polytechnic University.

Ivan A. Rozaev, assistant, National Research Tomsk Polytechnic University.
45. Mordvinov V.A., Turbakov M.S. K metodike vybora elektrotsentrobezhnyh nasosov pri ekspluatatsii neftedobyvayushchikh skvazhin [0n the methodology for selection of electric centrifugal pumps during the operation of oil wells]. Vestnik Permskogo gosudarstvennogo tekhnicheskogo universiteta. Geologiya, geoinformacionnye sistemy, gorno-neftyanoe delo, 2009, vol. 8, no. 4, pp. 59-67.

46. Vdovin V.V., Visloguzov D.P., Clan V.A., Kotin D.A., Pankratov V.V., Smetannikov A.V. Control algorithms and stability of direct current backup power supply for a frequency-controlled electric drive. Russian Electrical Engineering, 2015, vol. 86, Iss. 8, pp. 485-489. D0I: 10.3103/S106837121508012X.

47. Nos 0.V., Volkov V.Y., Klan V.A. Vector control for an AC drive with a multilevel frequency converter based on an N-bridge. Russian Electrical Engineering, 2019, vol. 90. no. 4, pp. 304-309.

Received: 30 September 2019. 